\title{
Design and Fabrication of a Novel Elastomers Material Testing System
}

\author{
Yen-Feng Cheng ${ }^{*}$, Pei-Chung Lin, Kuo-Shen Chen, Ching-Huei Chu \\ Department of Mechanical Engineering, National Cheng-Kung University, \\ No.1, Daxue Rd., East Dist., Tainan City 701, Taiwan (R.O.C.) \\ *Corresponding Author: happywego318@gmail.com
}

\begin{abstract}
In this work, a novel biaxial rubber bearing test system, as well as essential material characterization results, are realized and presented. This system integrates a voice coil actuator, a capacitive displacement sensor, and a load cell to perform compression tests of elastomers in both static and dynamic manners. On the other hand, the torsional degree of freedom is realized by a rotational stage, a torque sensor, and a wiimote camera. A simple but effective temperature controlling unit is also implemented for material testing between room temperature and $80^{\circ} \mathrm{C}$. Essential characterization on the compression and shear stiffness of PDMS and Dow 3310 with different shape factors at different temperature, as well as the influence of actuating frequencies, are performed for demonstrating the performance of the system.
\end{abstract}

Keywords: Bi-axial testing system, DMA, rubber characterization.

\section{Introduction}

Bearings are important machine elements for virtually all movable machinery. Laminate elastomer bearings are an alternative that can be designed and fabricated in a compact form with low $\operatorname{cost}^{(1)}$. One particular feature of elastomer bearing is the stiffness anisotropy for bonded elastomer $\operatorname{pads}^{(2,(3)}$. This implies that the compression (or radial) direction can be fully constrained while the shear (or axial) direction is still compliant for allowing motion by simply adjusting the extent of pre-compression ${ }^{(4)}$. In comparison with metal flexures commonly used for precision machinery, the elastomer bearing is more versatile and less complicated with lower manufacturing cost. As a result, it is important to perform accurate mechanical characterization of elastomer elements for machine design optimization.
Previous elastomer mechanical characterization schemes are usually bulky, slow and not fully automation such as the Yersley Oscillograph method ${ }^{(5)}$, standard torsional testing methods ${ }^{(6)}$. Although the above-mentioned schemes are well-fitted for traditional applications, they may not be adequate for applications required miniaturized size and high operating bandwidth. Consequently, Barton of MIT proposed a novel elastomer characterization system based on voice motor actuation for testing both the compression and shear stiffness from near DC to $3 \mathrm{k} \mathrm{Hz}^{(7)}$ as an auxiliary apparatus for developing their tool servo systems. Although it demonstrated high bandwidth and accurate deformation measurement, the lack of force sensing and temperature control actually made the test results suspicious. Meanwhile, although its modular design provided convenient in performing either compression or shear tests using the same system, the coupling or bi-axial effects cannot be examined. In this work, based on the work of $\operatorname{Barton}^{(7)}$, a more sophisticated elastomer stiffness characterization system is proposed and realized for biaxial actuation. This system integrates a voice coil actuator, a capacitive displacement sensor, and a load cell to perform compression tests of elastomers in both static and dynamic manners. On the other hand, the torsional degree of freedom is realized by a rotational stage, a torque sensor, and a wiimote camera. With simple while effective temperature controlling unit, this system can perform biaxial testing up to $80^{\circ} \mathrm{C}$ and actuating bandwidth approximately $100 \mathrm{~Hz}$. This system enables various material properties and bearing stiffness characterization in both compression and shear directions for supporting subsequent rubber bearing designs for precision motion systems.

The remainder of this paper presents the design, testing, and control of the stage in detail. A brief description on the stage conceptual design is outlined in Chapter 2 and followed by the detail description in system realization presented in 
Chapter 3. Associated specimen fabrication using PDMS and RTV 3110 as the primary testing materials for elucidating the applicability of the system is then described in Chapter 4. With the specimen ready, the system performances are then evaluated in Chapter 5 and followed by essential material testing addressed in Chapter 6 . Finally, Chapter 7 briefly discusses the current results, provides future perspective, and concludes this work.

\section{Conceptual design}

Before constructing the testing system, a conceptual design flow must be outlined for clarifying the complex issues and helping the system setup. To fit the most needs in precision machine design, the designed capacity in force measurement is $100 \mathrm{~N}$ and the maximum temperature of the system is $80{ }^{\circ} \mathrm{C}$. The conceptual design process of this system is shown in Figure 1. There are several key subsystems in this system including the temperature control, mechanisms and fixtures as well as the sensors and actuators. With these subsystems, the biaxial test system can perform tests in compression, stress relaxation, creep and dynamic mechanical analysis.

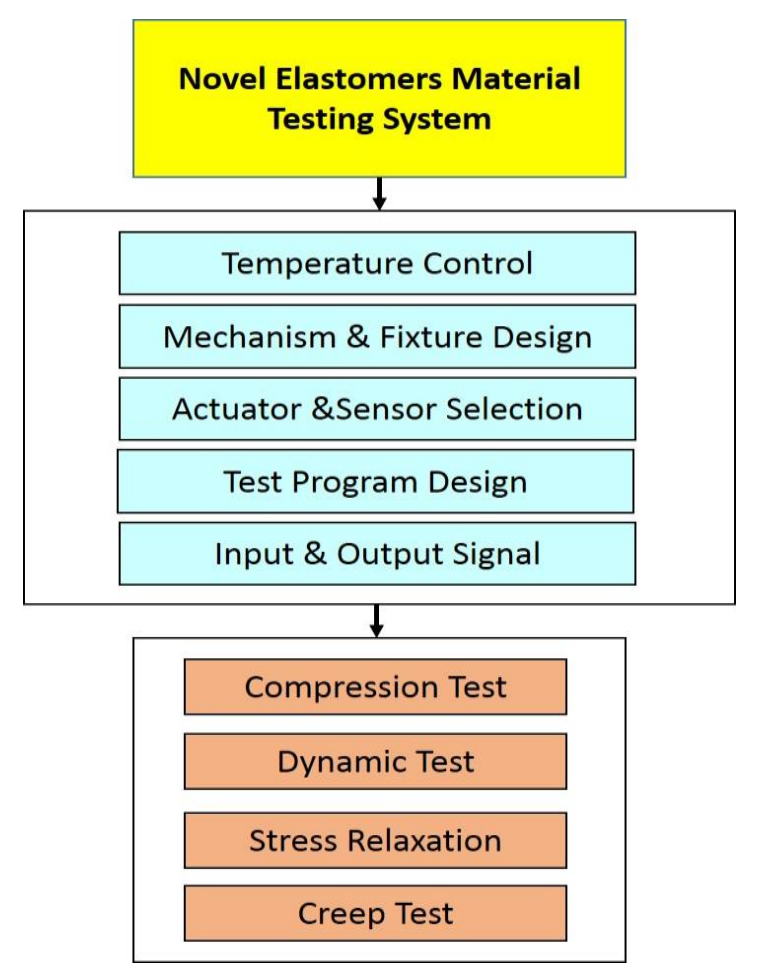

Fig. 1. The conceptual design flow chart

\section{Biaxial Test System}

\subsection{Structure and Actuator}

This mechanism of the system is shown in Figure 2. The structure consists of support columns, lift stage, and linear guide mounted on an optical table for stable and smooth operation, as well as for rejecting possible environmental disturbances. The lift stage can offer the movement in $\mathrm{Z}$ axis direction (perpendicular to horizontal plane) and the linear guide can limit the movement in $\mathrm{X}$ and $\mathrm{Y}$ directions.

Voice coils motor has the advantages of large stroke and high operation bandwidth ${ }^{(8)}$ and was chosen as the primary actuator in this work. A cylindrical voice coil motor (SMM AVM40-20) with a stroke of $20 \mathrm{~mm}$ and a forcing capacity of $58.1 \mathrm{~N}$ is finally selected. On the other hand, this system also uses a motorized rotation stage as the rotation actuator (SIGMAKOKI SGSP-60YAW-0B). It provides the rotation angle of 360 degrees and the maximum rotating speed of 30 degrees per second.

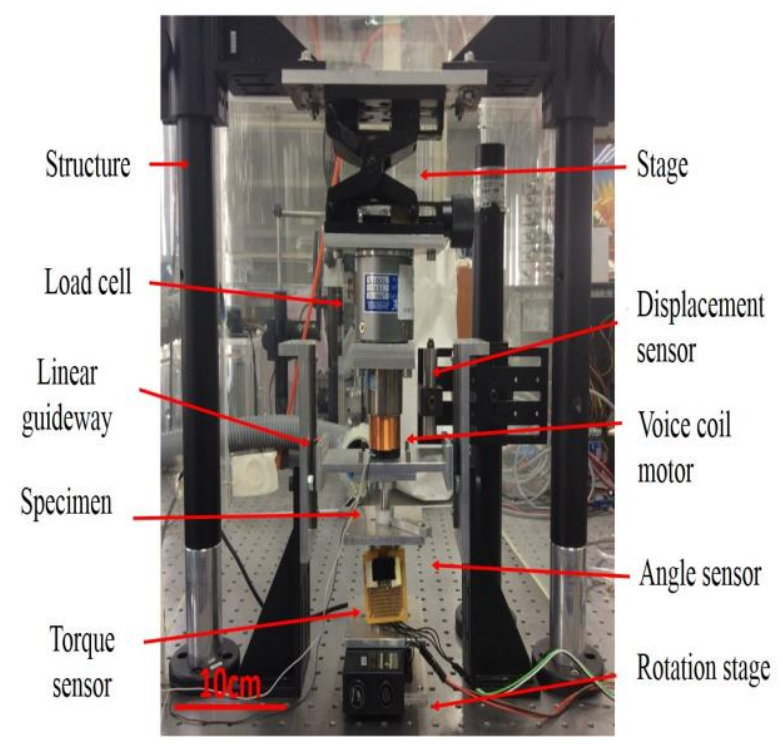

Fig. 2. A picture to show the structure of the biaxial testing system

\subsection{Sensor}

In the preliminary design of the system ${ }^{(8)}$, this system chose two kinds of displacement sensor for different testing ranges. A laser displacement sensor (MICROEPSILON optoNCDTILD 1400-10) with range of $10 \mathrm{~mm}$ and a resolution of $2 \mu \mathrm{m}$, is used for characterizing larger or less stiff specimens. On the other hand, a capacitive displacement sensor (MTI ASP -50 CTA) with sensing range of $1.2 \mathrm{~mm}$ and a resolution of $0.03 \mu \mathrm{m}$ is used for 
testing miniature or high stiffness specimens.

In previous work ${ }^{(7)}$, the actuating force was converted by monitoring the applied current. The results of such an indirect measurement, although simple in principle and can simplify the design, is actually suspicious since the transducer constant of the voice coil is actually not a constant and strongly depends on the system temperature. Without appropriate force measurement, the test results from $^{(7)}$ is questionable. For addressing this issue, this work chooses a load cell as the force sensor that can measure the force directly (ESENSE LRM-10) with a measurement range of $100 \mathrm{~N}$. Load cell is in serial connection with the voice coil motor for direct measurement.

Meanwhile, for monitoring the applied torque acting on specimens, a (ESENSE T-1) torque sensor is also integrated in this system shown in Figure 3. The measurement range of the torque sensor is $1 \mathrm{Nm}$. On the other hand, this work also adds a wiimote camera widely used in indoor localization ${ }^{(9)}$ (also shown in Figure 3) as the angular sensor. Wiimote camera can capture position data of an infrared source and the data can be converted into rotating angles using geometry relations subsequently. With such a setup, a true bi-axial testing system is finally realized.

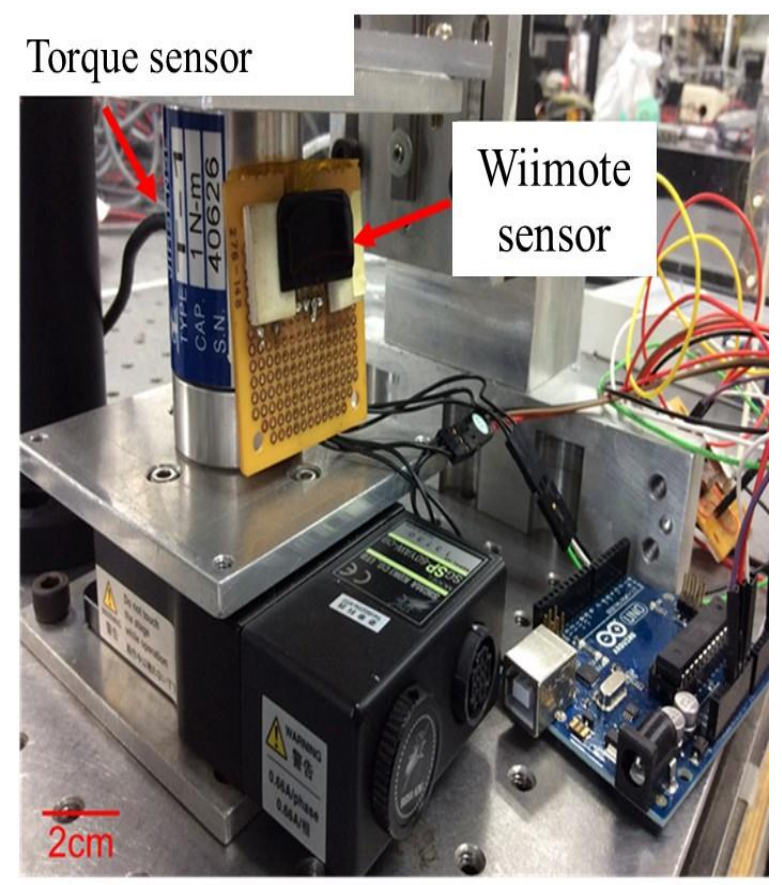

Fig. 3. Torque Sensor \& Wiimote camera for rotational degree of freedom monitoring

\subsection{Temperature Control System}

The mechanical properties of rubbers are sensitive to temperature variations. Therefore, a temperature control environment is required. To conduct experiments at desired temperatures, a small cabin made of transparent acrylic box is served as the temperature control unit. In together with necessary heating and ventilating, as well as the temperature controller and sensors, the test can be performed in the temperature range between room temperature and $80^{\circ} \mathrm{C}$ with accuracy level of $\pm 1^{\circ} \mathrm{C}$.

\section{Material Specimen Preparation}

In this work, the polydimethylsiloxane (PDMS) and RTV 3110 (Dow Corning ${ }^{\circledR}$ company) are chosen as the rubber in specimens. PDMS is a common viscoelastic material widely used in biomedical $\operatorname{MEMS}^{(10)}$ or soft lithography in nanoimprinting process ${ }^{(11)}$. On the other hand, RTV 3110 is often used in electronic packaging or sealants ${ }^{(12)}$ and has been used as a rubber bearing material ${ }^{(13)}$. Both of them are formed by mixing the main liquid primers with their solidifying agents with specific proportion (approximately 10:1). Notice that the stirring process would generate bubbles in the rubber and they must be removed under certain vacuum conditions since these bubbles would cause voids and the compressibility of the structure could be changed. Figure 4 shows the cylindrical rubber specimen with $10 \mathrm{~mm}$ in both radius and thickness. This achieves a corresponding shape factor of 1. By designing fixtures with different radius and thickness combination, specimens with desired shape factor combinations are accomplished through the casting and curing process.

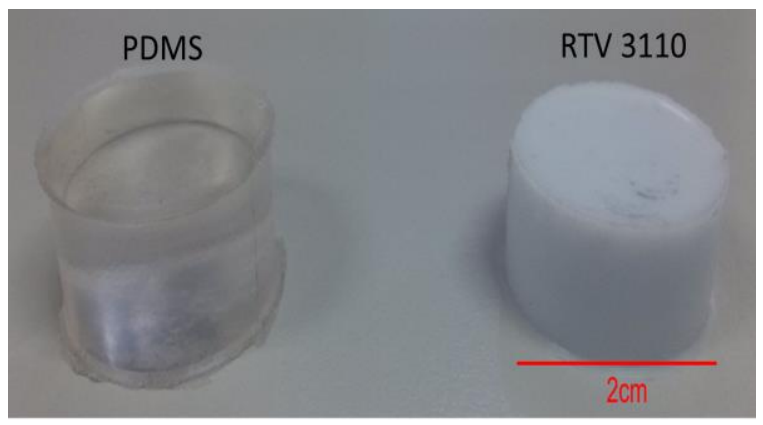

Fig. 4. PDMS and RTV 3110 specimens, shape factor $=1$

\section{System Integration and Performance Test}

After finishing the construction of each subsystem and assembly, the testing system is then subjected to several tests for evaluating its performance before conducting the subsequent material characterization. The integration and performance tests are described below. 


\subsection{System Stiffness Characterization}

The first issue to be evaluated is the stiffness of the system. Unlike Barton's work ${ }^{(7)}$, where the measurement is independent to the structure compliance, the stiffness of this system indeed influence the test results and must be precharacterized due to the consideration of adding a load cell and the placement of the displacement sensor. For example, during compression testing, both the specimen and the structure system will deform under the loading since they are connected in series. Thus the structural loop and the measurement loop become coupled together and the structure stiffness of the testing system must be determined in prior. By conducting essential characterization, the system stiffness, $k_{\text {system }}$, is determined as $148 \mathrm{~N} / \mathrm{mm}$. To reduce the error, the specimen stiffness should be much less than $k_{\text {system }}$. The measured stiffness, $k_{\text {total }}$, and the actual specimen stiffness, $k_{\text {sample }}$, have the following relation

$$
k_{\text {sample }}=\frac{k_{\text {total }} \times k_{\text {system }}}{k_{\text {system }}-k_{\text {total }}}
$$

One can extract the structure stiffness from the measured data to obtain the final result by Eq. (1).

\subsection{Natural Frequency and Bandwidth}

A system with a capability in high testing bandwidth is desired since this would make the testing become more versatile. To increase the bandwidth, the most direct manner is to increase the $1^{\text {st }}$ natural frequency of the system. By performing spectrum analysis during operation, we have observed a resonance peak at $38 \mathrm{~Hz}$. Through careful investigation, it is found that this peak is actually due to the structure compliance of the specimen, not the testing system. For example, as shown in Figure 5, this resonance frequency increases in square root manner with the specimen stiffness and this is a strong indication for supporting the above argument. This investigation suggests that specimens with larger shape factor should be used for characterizing the material properties at higher actuating frequencies.

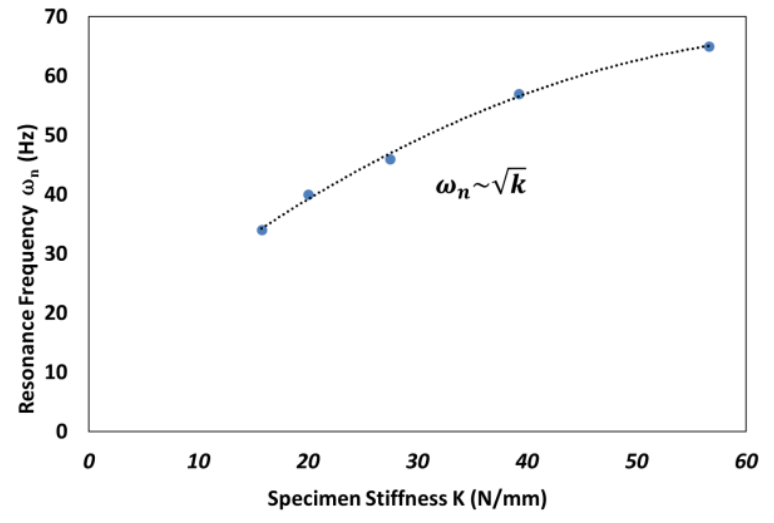

Fig. 5. The relation between the rubber stiffness and the $1^{\text {st }}$ resonance frequency of the testing system.

\subsection{Temperature Control Performance}

In order to test the performance of the temperature control environment, the system is heated up to $65^{\circ} \mathrm{C}$. In our previous design, without careful preventing heat loss, it requires near 3000 s to raise the system temperature to this level and the equivalent time constant is approximately $600 \mathrm{~s}$. With adding necessary shielding and hot air circulation, as well as a halogen lamp as the auxiliary thermal source (shown in Figures 6 and 7), the corresponding settling time and time constant are reduced to $1500 \mathrm{~s}$ and $200 \mathrm{~s}$, respectively. From the testing result shown in Figure 8, it is concluded that the testing system can achieve a temperature control in a reasonable time scale. The accuracy, as mentioned previously, is approximately $\pm 1^{\circ} \mathrm{C}$.

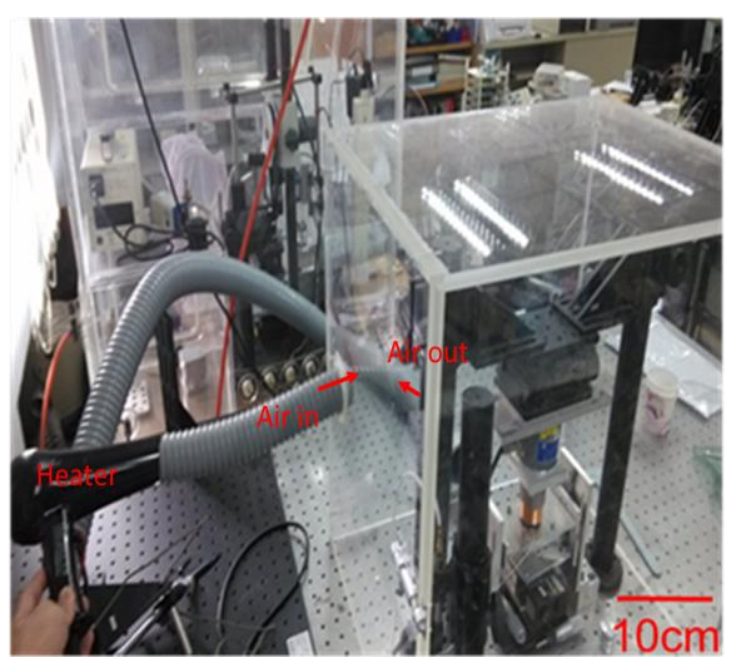

Fig. 6. The temperature controlled unit with air circulation 


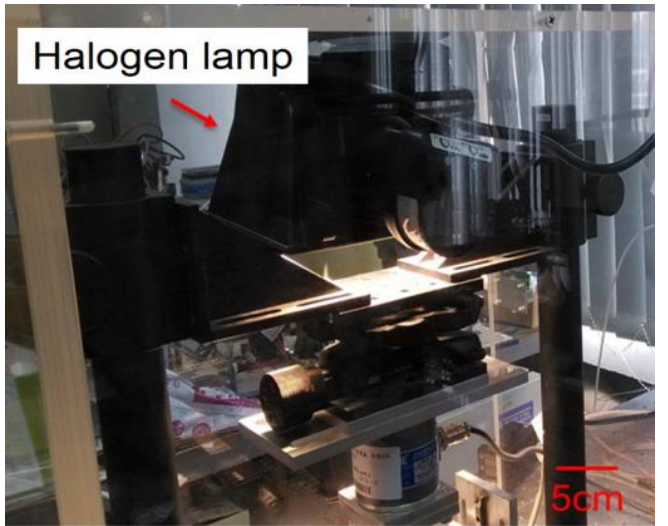

Fig. 7. Halogen lamp as the auxiliary heat resource

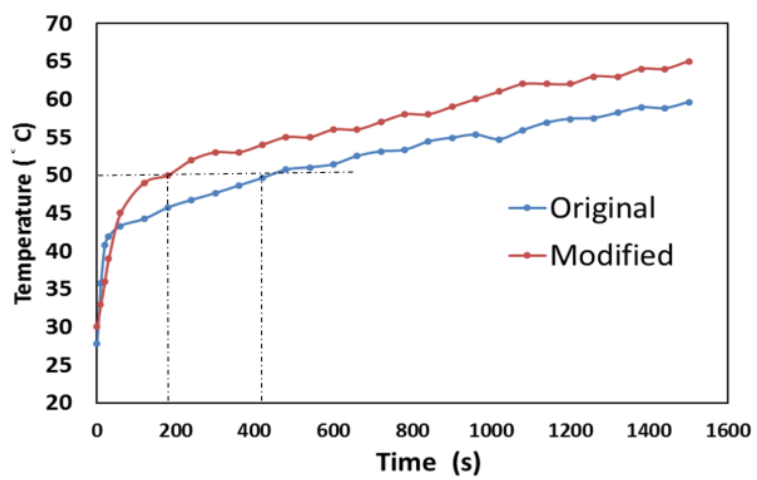

Fig. 8. Heating histories of different designs

\section{Primary Material Testing Results}

In this section, primary material characterization results of PDMS and RTV3310 are presented for demonstrating the applicability of the system.

\subsection{Rubber Compression Test}

Compression test is a common material test scheme and treated as the most important tests for rubber bearing characterization. The compression modulus obtained from this test could provide the deformation estimation of rubber bearing subjected to radial type loading. Meanwhile, due to their viscoelastic feature, the stiffness of rubber bearings is expected to be frequency-dependent. The testing should be performed under dynamic environment with different actuations frequencies ${ }^{(14)}$. A function generator provides a fixes sinusoidal input signal at different frequencies to actuate the system and both the loading and deformation signals are collected for calculating the variations of the stiffness of specimens. Meanwhile, the bearing capability also largely depends on the shape factor thus the behavior of rubber structures with different shape factors should be performed. Finally, as already mentioned above, the mechanical behavior of rubber materials is extremely sensitive to the operating temperature and the effect of temperature should also be evaluated.

\section{- Effect of actuating frequencies}

Figure 9 is the room temperature compression test results of 3110 RTV rubber specimen with a shape factor of 0.5 at various actuating frequencies. It can be seen that the stiffness increases with the actuating frequency. This agrees with the general tendency of rubber materials. The stiffness varies from $25.1 \mathrm{~N} / \mathrm{mm}$ at very low actuating frequency (i.e., $0.5 \mathrm{~Hz}$ ) to $45.7 \mathrm{~N} / \mathrm{mm}$ at $5 \mathrm{~Hz}$. Meanwhile, by converting the deformation to compression ratio and the reaction force to nominal stress, the equivalent compression modulus varies from $0.8 \mathrm{MPa}$ to $1.5 \mathrm{MPa}$.

\section{- Effect of shape factors}

RTV 3310 specimens with shape factors of $0.25,0.5$, 1 , and 2 are chosen for compression test for examining the effect of shape factors at room temperature with an actuating frequency of $1 \mathrm{~Hz}$, the test results are shown in Figure 10. The specimen becomes stiffer at larger shape factor. The compression modulus, as shown in Figure 11, increases with the shape factor.

\section{- Effect of testing temperature}

Finally, the effect of temperature is also characterized. RTV 3110 specimens with a shape factor of 0.5 are used to carry the experiments between room temperature and $60^{\circ} \mathrm{C}$ with an actuating frequency of $1 \mathrm{~Hz}$. The test results are shown in Figure 12. It can be seen that the specimen becomes more compliant as the temperature increases. The equivalent compression modulus decreases from 1.82MPa at room temperature to $1.19 \mathrm{MPa}$ at $60^{\circ} \mathrm{C}$.

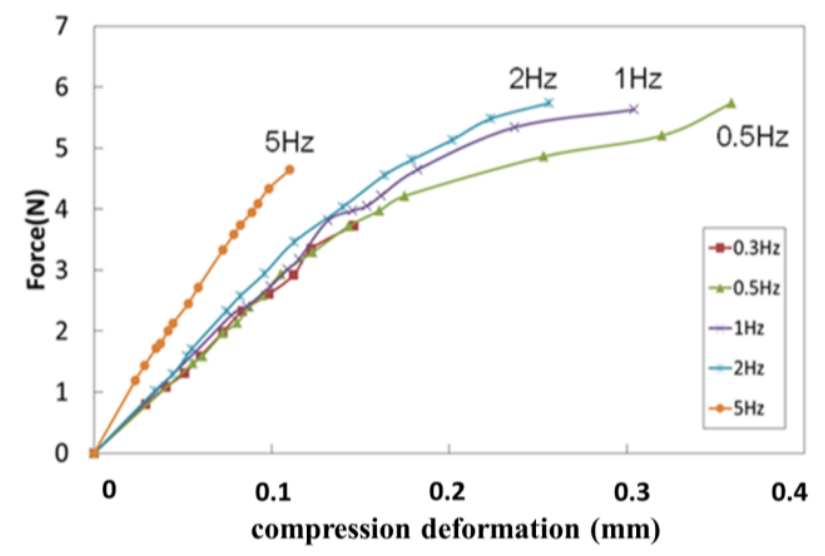

Fig. 9. The relation between force and compression deformation of the 3110 RTV Rubber in different frequency and shape $=0.5$. 


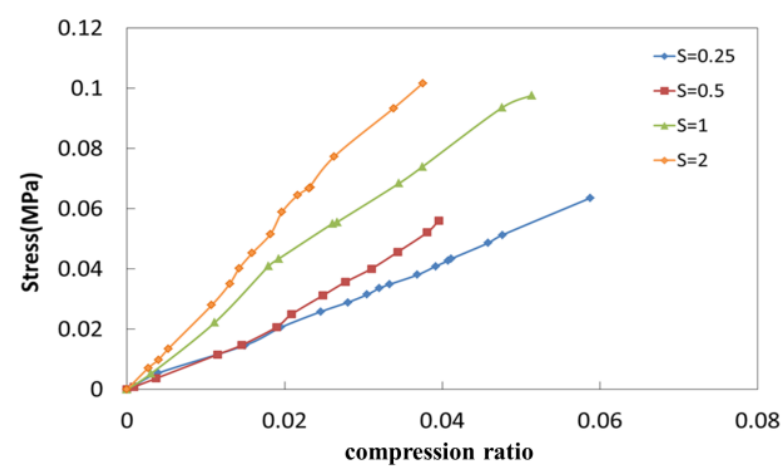

Fig. 10. The relation between stress and compression ratio of the 3110 RTV Rubber in different shape factor

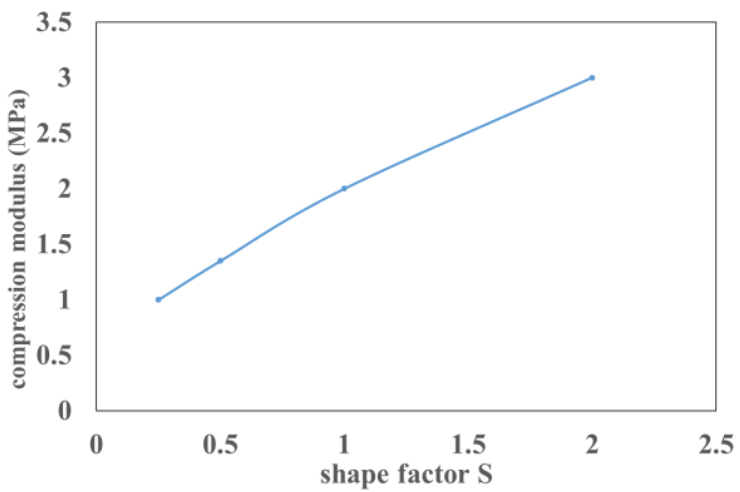

Fig. 11. The relation between compression modulus and shape factor of the 3110 RTV Rubber

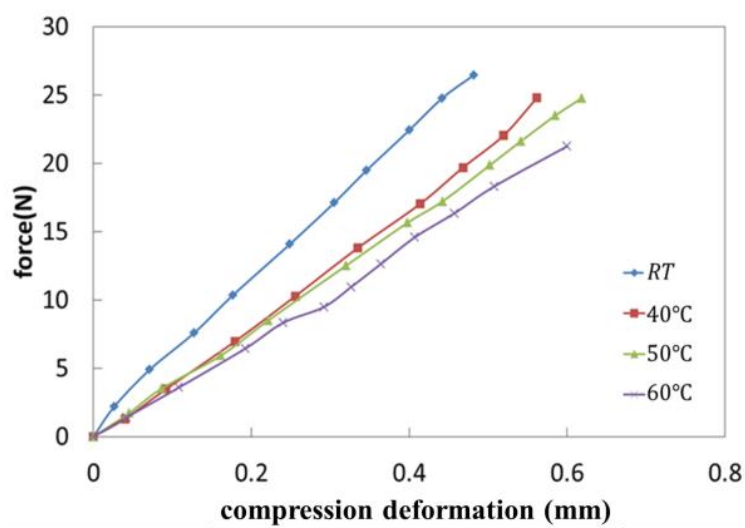

Fig. 12. The relation between force and compression deformation of the 3110 RTV Rubber in different environment temperature

\subsection{Rubber Shear Test}

After finishing the compression test, it is also worth to demonstrating the capability of conducting shear test by the designed system. Shear test is another important testing for evaluating the performance of a rubber bearing. It actually represents the compliance of the moving direction. In together with the compression test results, the most critical parameter, compression to shear stiffness ratio, can be obtained for evaluating the performance of a rubber bearing.
Meanwhile, in order to discuss the torsion-carry capability, it is also worth to perform torsional test by this biaxial system. As schematically shown in Figure 13, an annular shape PDMS specimen with both inner and outer edge fully constrained is designed to perform both shear and torsional tests. With a length of $10 \mathrm{~mm}$, the thickness $\mathrm{d}$ of PDMS specimens varies as 5,10 , and $15 \mathrm{~mm}$ for carrying the test. The torsional test results are shown in Figure 14. For the same rotating angle, a specimen with smaller thickness implies that a larger rate of twist. Consequently, it requires a larger applied torque.

Finally, the effect of pre-twisting on the shear stiffness of PDMS specimens is also characterized. This is for examining the performance variation of twisted rubber bearing due to possible misalignment during assembly. In addition, it also serves as the demonstration example for true biaxial test. The shear stiffness of the specimen with pretwisting of 5, 10,15 , and 20 degrees is characterized with a shearing frequency of $1 \mathrm{~Hz}$. The results are shown in Figure 15 and the results indicate that the shear stiffness slightly reduced as the twisting angle increases. More detailed experiments are currently planned for completely characterizing this issue.

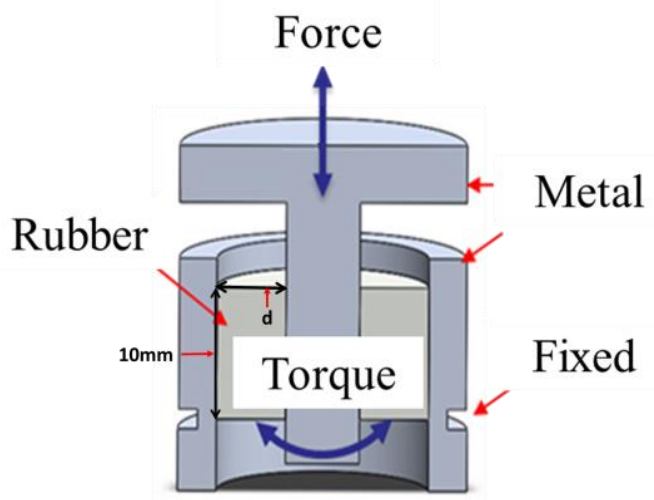

Fig. 13. The section of the biaxial fixture design

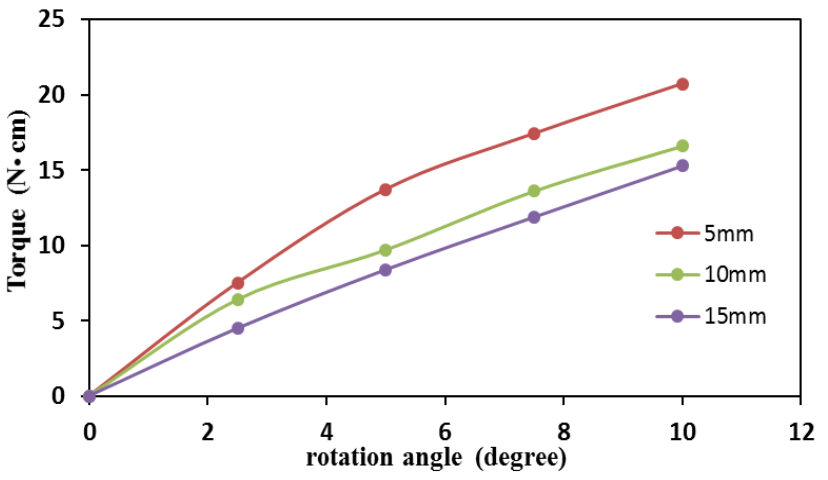

Fig. 14. The relation between torque and angle in different rubber width of the PDMS 


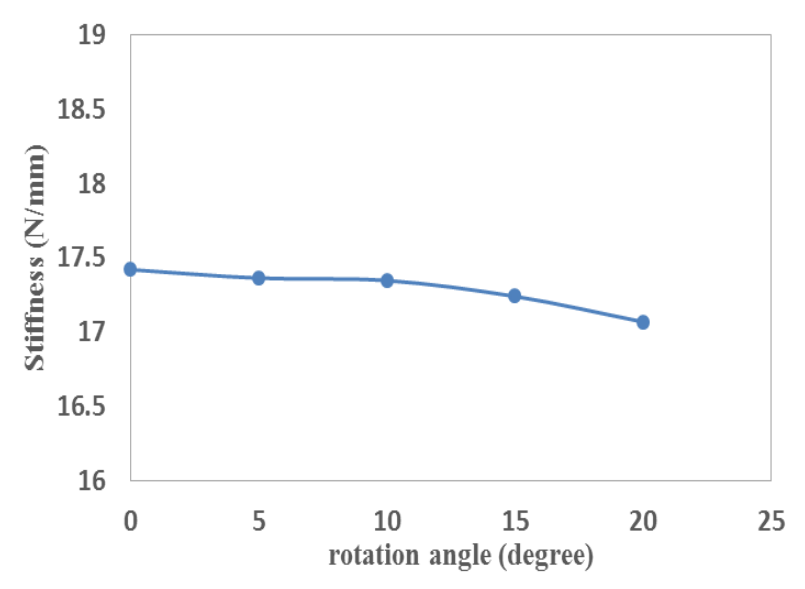

Fig. 8 The relation between stiffness and rotation angle of the PDMS

\section{Conclusions}

Rubber bearings have been introduced into precision machine design for improving the system performance recently. However, its stiffness anisotropy and viscoelastic nature should be accurate characterized. Although several testing systems have been developed previously, they may not be adequate for providing services in rubber material characterization for precision engineering. In this work, a novel elastomer material characterization system is designed and realized based on Barton's research. The modular design approach allows us to perform both compression and shear tests by simply change a particular module. By using a voice coil actuator as the actuator and a capacitance probe as the position sensor, this design can have high bandwidth and fine spatial resolution. Both are vital for conducting elastomer characterizations at small scale. Finally, several elastomer characterization activities have been conducted to demonstrate the advantage and possible applications of the test system for elastomer static and dynamic characterizations. We believe this work provides contributions in both mechatronics design and material characterization communities. In the future, this system can also be possible to characterize the long term reliability, such as fatigue, of elastomer bearings in a reasonable time.

\section{Acknowledgment}

This work is supported by Ministry of Science Technology (MOST) of Taiwan, ROC under the contract number 101-2221-E-006-032-MY3. The assistance and suggestions from Dr. K-S Ou in the early stage of this work is also greatly appreciated.

\section{References}

(1) E. I. Rivin, "Properties and prospective applications of ultra thin layered rubber-metal laminates for limited travel bearings", Tribology International, vol. 16, no. 1, pp. 17-25, Feb. 1983.

(2) J. Kelly and D. A. Konstantinidis, Mechanics of rubber bearings for seismic and vibration isolation, Wiley, 2011.

(3) E. I. Rivin. Stiffness and damping in mechanical design, Marcel Dekker, 1999

(4) P. B. Lindley, "Compression module for blocks of soft elastic material bonded to rigid end plates," Journal of Strain Analysis, vol. 14, pp. 11-16, 1979.

(5) ASTM D 945-06, "Standard test methods for rubber properties in compression or shear (mechanical oscillograph)," ASTM, 2006.

(6) P. A. Hilton Ltd., HSM43 Torsion testing machine, http://www.p-a-hilton.co.uk/products/HSM43-TorsionTesting-Machine-100Nm

(7) A. E. Barton-Martinelli, Rubber bearings for precision positioning systems, Master Thesis, Department of Mechanical Engineering, Massachusetts Institute of Technology, USA, 2005.

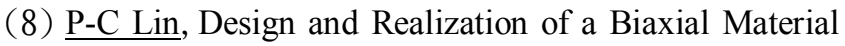
Characterization System for Elastomer Bearing Applications, Master Thesis, Department of Mechanical Engineering, National Cheng Kung University, R.O.C.

(9) D. Gu and K-S Chen, "Design and Performance Evaluation of Wiimote-Based Two-Dimensional Indoor Localization Systems for Intelligent Living Applications" Measurement, Vol. 66, pp. 95-108, 2015.

(10) J. M. Engel et al., "Multi-layer embedment of conductive and non-conductive PDMS for all-elastomer MEMS," In Proceeding of 2006 Solid-State Sensor and Actuator Workshop, p. 4-8, Hilton Head, SC, USA, 2006.

(11)X-M Zhao and Y. Xia, and G. M. Whitesides, "Soft lithographic methods for nano-fabrication," J. Mater. Chem., Vol. 7, pp. 1069-1074, 1997.

(12) J. M. Booe, "Elastomeric matrix with alkaline earth oxide," U.S. Patent no. 4,081,397, 1978.

(13) D. P. Cuff., Electromagnetic nanopositioner, M. S. thesis, Department of Mechanical Engineering, Massachusetts Institute of Technology, USA, 2006.

(14) K. P. Menard, Dynamic mechanical analysis: a practical introduction, CRC press LLC, 1999. 